\title{
Neuroimaging Predictors of Cognitive Impairment in Confluent White Matter Lesion: Volumetric Analyses of 99 Brain Regions
}

\author{
Vincent C.T. Mok ${ }^{a}$ Tianming Liu ${ }^{d, e}$ Wynnie W.M. Lam ${ }^{b}$ Adrian Wong ${ }^{a}$ \\ Xintao Hu ${ }^{f}$ Lei Guo ${ }^{f}$ Xiang Yan Chen ${ }^{a}$ Wai Kwong Tang ${ }^{c}$ Ka Sing Wong ${ }^{a}$ \\ Stephen Wong d, e \\ Departments of a Medicine and Therapeutics, ${ }^{\mathrm{b}}$ Radiology and Organ Imaging, and ${ }^{\mathrm{C}}$ Psychiatry, Chinese University

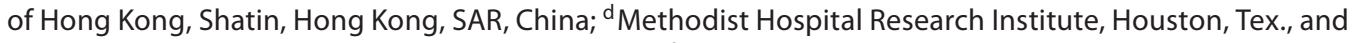 \\ 'Weill Cornell Medical College, New York, N.Y., USA; fSchool of Automation, Northwestern Polytechnic University, \\ Xi'an, China
}

\section{Key Words \\ Confluent white matter lesion · Neuroimaging predictors • Cognitive impairment}

\begin{abstract}
Background: Although confluent white matter lesion (WML) is associated with cognitive impairment, the mechanism explaining this association is controversial. We aimed to investigate comprehensively the MRI predictors of cognitive impairment in confluent WML. Methods: Among 45 lacunar stroke patients who had confluent WML, we evaluated the association of executive function [Mattis Dementia Rating Scale - Initiation/Perseveration subscale (MDRS I/P)] and global cognition [Mini-Mental State Examination (MMSE)] with the volume of WML, measures of lacunes and microbleeds, and the volumes of 99 other specific brain regions. Results: Regression analyses showed that WML volume predicted performance on the MDRS I/P $(\beta=-0.34, p=0.016)$ independent of age. Volumes of cortical gray matter (cGM; $\beta=0.41, p=0.003)$, the lateral fronto-orbital gyrus $(\beta=0.38$, $p=0.01)$, superior frontal gyrus $(\beta=0.29, p=0.04)$, lateral ventricle $(\beta=-0.30, p=0.04)$, and posterior limb of the in-
\end{abstract}

\section{KARGER}

Fax +4161306 1234

E-Mail karger@karger.ch

www.karger.com

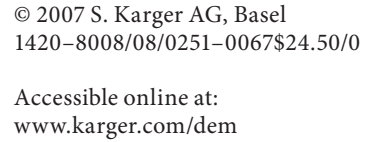

ternal capsule $(\beta=0.43, p=0.002)$ predicted MDRS I/P performance independent of WML volume. Volumes of $c G M$, and the lateral fronto-orbital gyrus predicted MMSE performance as well. Conclusion: Atrophy along the frontosubcortical pathways and CGM predict cognition in confluent WML independent of WML volume. Copyright $\odot 2007$ S. Karger AG, Basel

Although confluent white matter lesion (WML), or leukoaraiosis, can be associated with multiple causes, e.g. demyelination, cerebral amyloid angiopathy, or cerebral autosomal dominant arteriopathy with subcortical infarcts and leukoencephalopathy, by far the commonest cause is related to diffuse arteriosclerotic small-vessel disease (SVD). Aging and traditional vascular risk factors, e.g. hypertension, are implicated in the development of diffuse arteriosclerotic SVD [1]. Prevalence of such age-related confluent WML reaches as high as $20 \%$ among elderly aged 65 or above [2].

Age-related confluent WML has long been associated with cognitive impairment. It has been considered the key substrate for subcortical vascular dementia, or Bins-
Vincent C.T. Mok

Department of Medicine and Therapeutics

The Chinese University of Hong Kong

Shatin, Hong Kong, SAR (China)

Tel. +852 2632 2195, Fax +852 2637 3852, E-Mail vctmok@cuhk.edu.hk 
wanger's disease $[3,4]$ and its presence is included as part of the imaging criterion for diagnosing vascular dementia or subcortical vascular dementia $[3,5]$. Longitudinal studies showed that progression of confluent WML is more rapid relative to that of punctuate WML [6] and WML progression parallels cognitive decline [7]. Authorities recently proposed that preventive trials in vascular cognitive impairment should target patients having confluent WML at baseline and its progression may be used as a surrogate marker $[6,8]$.

Albeit the evidence on the association between confluent WML and cognitive impairment seems strong, it has also been observed that confluent WML is not necessarily associated with cognitive impairment [9]. Other imaging variables may affect cognition as well. An early small series observed that among patients with severe WML, those with dementia had severer callosal atrophy than those without [10]. Recent studies on cerebral SVD focused on patients with lacunes and only a small proportion had severe WML. These studies showed that atrophy in cortical gray matter (cGM), the hippocampus, corpus callosum, or frontal lobe and the presence of thalamic lacunes are robust cognitive predictors [11-14]. Whether these imaging variables also affect cognition in confluent WML is unknown. To date, imaging study among those having confluent WML with a spectrum of cognitive impairment has been limited. In this study, we comprehensively searched more than 100 imaging variables for MRI predictors of cognitive impairment in confluent WML using a quantitative method.

\section{Methods}

\section{Patients}

Subjects were participants of the MRI substudy (clinical trial ID: CUHK_CCT00122) of the VITATOPS (Vitamins to Prevent Stroke) study [15] that evaluated homocysteine-lowering therapy in progression of confluent WML and cognition over 2 years among 100 ischemic Chinese stroke patients with confluent WML who were admitted to the acute stroke unit of a university-based hospital. We excluded patients with cognitive or physical impairment severe enough to prevent cognitive assessment. The ethics committee approved the study and all participants provided consent. The same neurologist (V.M.) screened for the presence of confluent WML on FLAIR MRI [16]. Among the 100 participants, we selected 45 patients who had lacunar stroke for the present study. The mean volume of WML $\left(41.2 \mathrm{~cm}^{3}\right)$ of these 45 patients was 30 times higher than that of our previous cohort with punctuate WML $\left(1.32 \mathrm{~cm}^{3}\right)$ [16]. We assessed stroke severity using the Oxford Handicap Scale (OHS) and recorded systolic blood pressure (SBP) and diastolic blood pressure (DBP) levels at 3 months after stroke.
Table 1. Imaging variables that were evaluated
Ischemia-related variables
1 Total volume of WML
2 Number and volume of lacunes: total; thalamus; basal ganglia; other regions
3 Number of microbleeds: total; basal ganglia; thalamus

\section{Atrophy-related variables}

1 Superior frontal gyrus

2 Middle frontal gyrus

3 Inferior frontal gyrus

4 Medial fronto-orbital gyrus

5 Lateral fronto-orbital gyrus

6 Precentral gyrus

7 Postcentral gyrus

8 Supramarginal gyrus

9 Superior parietal lobule

10 Precuneus

11 Angular gyrus

12 Cuneus

13 Lingual gyrus

14 Superior occipital gyrus

15 Middle occipital gyrus

16 Inferior occipital gyrus

17 Medial occipitotemporal gyrus

18 Lateral occipitotemporal gyrus

19 Occipital pole

20 Insula

21 Superior temporal gyrus

22 Middle temporal gyrus

23 Inferior temporal gyrus

24 Temporal pole

25 Parahippocampal cortex

26 Hippocampal formation

27 Entorhinal cortex

\author{
28 Perirhinal cortex \\ 29 Uncus \\ 30 Cingulate region \\ 31 Corpus callosum* \\ 32 Globus palladus \\ 33 Caudate nucleus \\ 34 Putamen \\ 35 Thalamus \\ 36 Anterior limb of internal capsule \\ 37 Posterior limb of internal capsule \\ 38 Subthalamic nucleus \\ 39 Amygdala \\ 40 Nucleus accumbens \\ 41 Fornix \\ 42 Frontal lobe WM \\ 43 Parietal lobe WM \\ 44 Temporal lobe WM \\ 45 Occipital lobe WM \\ 46 Cerebellum \\ 47 Brain stem* \\ 48 Lateral ventricle \\ 49 Third ventricle* \\ 50 Fourth ventricle* \\ 51 Subarachnoid CSF* \\ 52 Total cGM* \\ 53 Total brain volume ${ }^{*}$
}

Volumes of both sides, i.e. left and right, were obtained for all the above regions except those marked with an asterisk.
Cognitive Assessment

Methods of cognitive assessment were described previously [17]. In brief, a psychologist (A.W.) administered the Chinese version of the Mattis Dementia Rating Scale - Initiation/Perseveration subscale (MDRS I/P) [18] and the Mini-Mental State Examination (MMSE) [19] to all patients at 3 months after stroke. The MMSE and MDRS I/P are brief measures of global cognition and executive function, respectively. We used the Clinical Dementia Rating Scale (CDR) [20] and the Chinese version of the Lawton Instrumental Activities of Daily Living (IADL) scale [21] to grade the severity of cognitive and functional impairment. The score for each of the 8 IADL items ranges from 0 to 3, with a lower score representing better function. As some items in the IADL scale were not scored because they might not be applicable to all patients owing to personal habits or motor impairment, we took the average of all scored items as the final IADL score. In grading impairment in each of the categories, care was taken to grade only those impairments that were attributed to cognitive symptoms, and not to motor or mood disturbances. 
Table 2. Patient characteristics $(\mathrm{n}=45)$

\begin{tabular}{lc}
\hline Mean age, years & $74.6(8.3)$ \\
Female & $18(40 \%)$ \\
Mean years of education & $4.2(3.5)$ \\
Hypertension & $39(86.7 \%)$ \\
Diabetes mellitus & $15(33.3 \%)$ \\
Smoker & $24(53.3 \%)$ \\
Mean score of OHS & $1.7(1.3)$ \\
Mean score of MDRS I/P & $26.8(6.8)$ \\
$\quad$ Range & $3-37$ \\
Mean MMSE score & $23.0(4.3)$ \\
$\quad$ Range & $14-29$ \\
Mean IADL (average of all individual scored items) & $1.1(1.0)$ \\
$\quad$ Range & $0.0-2.6$ \\
CDR & \\
$\quad$ (number of patients having ratings of $0 / 0.5 / 1$ or 2$)$ & $12 / 30 / 3$ \\
Mean volume of WML, cm ${ }^{3}$ & $41.2(22.0)$ \\
$\quad$ Range & $6.1-104.7$ \\
Measures of lacunes & \\
$\quad$ Mean total number & $4.5(3.4)$ \\
$\quad$ Median total number & $3.0(4.0)$ \\
$\quad$ Mean total volume, $\mathrm{cm}^{3}$ & $0.7(0.6)$ \\
$\quad$ Median volume of thalamic lacunes, cm ${ }^{3}$ & $0.0(0.1)$ \\
Presence of thalamic lacune & $20(44.4 \%)$ \\
Measures of microbleeds & \\
$\quad$ Median total number & \\
Median number of thalamic/basal ganglia micro- & $1(12.8)$ \\
bleeds & $0.0(5.0)$ \\
$\quad$ Mean volume of cGM, cm ${ }^{3}$ & $395.0(50.1)$ \\
\hline
\end{tabular}

Figures in parentheses are standard deviations unless otherwise specified.

${ }^{1}$ One outlier with a total number of microbleeds of 133 was excluded.

\section{MRI Variables}

We performed brain MRI on subjects within 1 week of admission (table 1). The MRI examinations were done with a 1.5-tesla scanner (Sonata, Siemens Medical System, Erlangen, Germany).

Ischemia-Related Variables. Methods assessing the volume of WML and lacunes were described previously [13]. In brief, total volume $\left(\mathrm{cm}^{3}\right)$ of WML was measured with Easy Vision 4.3 on the FLAIR sequence using the semi-automated segmentation method by A.W. and C.X.Y. Microbleeds were defined as dot-like hypointensity on gradient recall echo $\mathrm{T}_{2}{ }^{*}$-weighted MRI [22] and were assessed by W.W.M.L. and V.M. Interrater reliability based on random selection of 7 films was excellent for both WML $(r=0.91)$ and microbleeds $(\mathrm{r}=0.97)$. All assessors were blinded to patients' cognitive data.

Atrophy-Related Variables. We applied the Oxford FSL tools (http://www.fmrib.ox.ac.uk/fsl/) for preprocessing and brain segmentation of the $\mathrm{T}_{1}$-weighted MR images. The Brain Extraction Tool was used to remove the noncerebral tissues such as bone or skin. Then the automated segmentation tool FAST [23] was used to segment the brain into gray matter, white matter, and cerebro- spinal fluid compartments. The hybrid volumetric and surface warping method [24] was then employed to automatically parcellate the structural brain MR image into a variety of regions. The evaluation and validation of the hybrid warping method were described previously [24]. After that, the subject's brain image was segmented into various regions (table 1) $[24,25]$. Meanwhile, we applied automatic tissue segmentation on the subject's structural MR image and used the resulting tissue maps to mask the automatically labeled structural MR image, generating the labeled regional maps. The scientists X.H. and T.L. performed the preprocessing and hybrid warping method, respectively.

\section{Statistical Analyses}

We first performed Pearson correlation of MDRS I/P and MMSE with each imaging variable (table 1 ). We then entered putative imaging variables that had significant Pearson correlation $(p<0.05)$ with cognitive performances into 2 regression models to find predictors of performance on the MDRS I/P and MMSE separately. In model 1 , age and education were adjusted. In model 2, age, education, and volume of WML were adjusted. For data that were not normally distributed, we performed square root transformation before entering them into the regression models. We also explored whether age, WML volume, SBP, and DBP predicted volumes of those cognitively relevant imaging predictors. To understand the relationship between cognitive performance and IADL, we performed regression analyses to explore the contribution of the OHS, MDRS I/P, and MMSE in predicting IADL.

\section{Results}

The characteristics of the 45 subjects are shown in table 2. The cohort's cognitive severity varied from 3 to 37 , 14 to 29 , and 0 to 2 for the scores of the MDRS I/P, MMSE, and CDR, respectively. Regression analyses revealed that among the OHS, MDRS I/P, and MMSE, only the OHS $(\beta=0.55, \mathrm{p}<0.001)$ and $\operatorname{MDRS} \mathrm{I} / \mathrm{P}(\beta=-0.37, \mathrm{p}=0.003)$ were significant predictors of IADL.

Regions with significant cognitive Pearson correlation are listed in table 3. Further regression analyses demonstrated that volume of WML predicted MDRS I/P ( $\beta=$ $-0.34, \mathrm{p}=0.016)$ but not MMSE performance. Volumes of cGM, the left lateral fronto-orbital gyrus, right superior frontal gyrus, left lateral ventricle, and right posterior limb of the internal capsule predicted MDRS I/P performance independent of WML volume (table 4a). Predictors of MMSE performance are shown in table $4 \mathrm{~b}$.

Among age, WML volume, SBP, and DBP, WML volume predicted volumes of the fronto-orbital gyri (left: $\beta=-0.27, p=0.01$; right: $\beta=-0.43, p=0.01)$ and left lateral ventricle $(\beta=0.39, p=0.01)$. Correlations of WML volume with the right superior frontal gyrus $(\beta=$ $-0.30, \mathrm{p}=0.07)$ and SBP with the volume of cGM almost 
Table 3. Variables with a significant Pearson correlation with cognitive performances

\begin{tabular}{|c|c|c|c|}
\hline Variables & & MDRS I/P & MMSE \\
\hline \multirow[t]{2}{*}{ Age } & $\mathrm{r}$ & -0.37 & -0.38 \\
\hline & $\mathrm{p}$ & 0.01 & 0.01 \\
\hline \multirow[t]{2}{*}{ Education } & $\mathrm{r}$ & - & 0.32 \\
\hline & $\mathrm{p}$ & - & 0.03 \\
\hline \multirow[t]{2}{*}{ cGM } & $\mathrm{r}$ & 0.49 & 0.54 \\
\hline & $\mathrm{p}$ & 0.0008 & 0.0001 \\
\hline \multirow[t]{2}{*}{ WML } & $\mathrm{r}$ & -0.34 & - \\
\hline & $\mathrm{p}$ & 0.02 & - \\
\hline \multirow[t]{2}{*}{ Thalamic lacune number } & $\mathrm{r}$ & - & 0.31 \\
\hline & p & - & 0.04 \\
\hline \multirow[t]{2}{*}{ Lateral ventricle left } & $\mathrm{r}$ & -0.46 & - \\
\hline & $\mathrm{p}$ & 0.002 & - \\
\hline \multirow[t]{2}{*}{ Lateral fronto-orbital gyrus right } & $\mathrm{r}$ & 0.31 & 0.32 \\
\hline & $\mathrm{p}$ & 0.04 & 0.03 \\
\hline \multirow[t]{2}{*}{ Lateral ventricle right } & $\mathrm{r}$ & -0.39 & - \\
\hline & $\mathrm{p}$ & 0.008 & - \\
\hline \multirow[t]{2}{*}{ Superior frontal gyrus right } & $\mathrm{r}$ & 0.35 & 0.33 \\
\hline & $\mathrm{p}$ & 0.02 & 0.03 \\
\hline \multirow[t]{2}{*}{ Globus palladus left } & $\mathrm{r}$ & 0.31 & - \\
\hline & $\mathrm{p}$ & 0.04 & - \\
\hline \multirow[t]{2}{*}{ Temporal pole right } & $\mathrm{r}$ & 0.33 & 0.41 \\
\hline & $\mathrm{p}$ & 0.03 & 0.005 \\
\hline \multirow[t]{2}{*}{ Posterior limb of the internal capsule } & $\mathrm{r}$ & 0.44 & 0.34 \\
\hline & $\mathrm{p}$ & 0.003 & 0.02 \\
\hline \multirow[t]{2}{*}{ Hippocampal formation right } & $\mathrm{r}$ & - & 0.46 \\
\hline & $\mathrm{p}$ & - & 0.002 \\
\hline \multirow[t]{2}{*}{ Lateral fronto-orbital gyrus left } & $\mathrm{r}$ & 0.36 & 0.33 \\
\hline & $\mathrm{p}$ & 0.02 & 0.03 \\
\hline \multirow[t]{2}{*}{ Postcentral gyrus right } & $\mathrm{r}$ & 0.36 & 0.31 \\
\hline & $\mathrm{p}$ & 0.01 & 0.041 \\
\hline \multirow[t]{2}{*}{ Middle occipital gyrus left } & $\mathrm{r}$ & - & 0.43 \\
\hline & $\mathrm{p}$ & - & 0.003 \\
\hline
\end{tabular}

reached statistical significance $(\beta=-0.29, p=0.059)$. None of the variables had a significant correlation with the volumes of the temporal pole, hippocampal formation, middle occipital gyrus, and posterior limb of the internal capsule.

\section{Discussion}

We analyzed the baseline MRI variables of participants of the VITATOPS study who had lacunar stroke and confluent WML. In these patients with confluent WML, apart from stroke severity, performance in the executive test also contributed to impairment in IADL. Note that performance in the MMSE had no significant
Table 4. Regression analyses

a Predictors of MDRS I/P performance

\begin{tabular}{lrlllll}
\hline & \multicolumn{2}{l}{ Model 1} & & \multicolumn{2}{l}{ Model 2 } \\
\cline { 2 - 3 } \cline { 6 - 7 } & \multicolumn{1}{c}{$\beta$} & $\mathrm{p}$ & & $\beta$ & $\mathrm{p}$ \\
\hline Age & -0.37 & 0.012 & & \\
WML & -0.34 & 0.02 & & \\
cGM & 0.41 & 0.01 & & 0.41 & 0.003 \\
Lateral ventricle left & -0.39 & 0.01 & & -0.30 & 0.04 \\
Lateral fronto-orbital gyrus right & 0.33 & 0.02 & & 0.25 & 0.08 \\
Lateral ventricle right & -0.34 & 0.02 & & -0.26 & 0.08 \\
Superior frontal gyrus right & 0.36 & 0.01 & & 0.29 & 0.04 \\
Globus palladus left & 0.24 & 0.1 & & 0.17 & 0.25 \\
Temporal pole right & 0.34 & 0.02 & & 0.27 & 0.06 \\
Posterior limb of the internal & & & & \\
$\quad$ capsule right & 0.36 & 0.02 & & 0.43 & 0.002 \\
Lateral fronto-orbital gyrus left & 0.46 & 0.01 & & 0.38 & 0.01 \\
Postcentral gyrus right & 0.28 & 0.08 & & 0.22 & 0.14 \\
\hline
\end{tabular}

b Predictors of MMSE performance

\begin{tabular}{lrlll}
\hline Age & -0.38 & 0.01 & & \\
Education & 0.32 & 0.03 & & \\
cGM & 0.45 & 0.001 & 0.45 & 0.001 \\
Thalamic lacune presence & 0.19 & 0.18 & 0.25 & 0.10 \\
Lateral fronto-orbital gyrus right & 0.33 & 0.02 & 0.34 & 0.02 \\
Superior frontal gyrus right & 0.27 & 0.05 & 0.27 & 0.06 \\
Temporal pole right & 0.41 & 0.002 & 0.41 & 0.003 \\
Posterior limb of the internal & & & & \\
$\quad$ capsule & 0.22 & 0.13 & 0.24 & 0.11 \\
Hippocampal formation right & 0.19 & 0.19 & 0.40 & 0.01 \\
Lateral fronto-orbital gyrus left & 0.39 & 0.004 & 0.43 & 0.004 \\
Postcentral gyrus right & 0.17 & 0.25 & 0.16 & 0.30 \\
Middle occipital gyrus left & 0.38 & 0.004 & 0.39 & 0.004 \\
& & & &
\end{tabular}

influence on IADL among our patients with confluent WML.

In this study, we employed a method that is capable of quantifying 99 brain regions to investigate the MRI predictors of cognitive impairment in confluent WML. To our knowledge, this is the most comprehensive study of this kind. Our study showed that even in patients with confluent WML, more diffuse WML was associated with greater executive impairment. This finding suggests that retarding progression of WML among those who already have confluent WML at baseline may still be relevant [6, 8]. Note also that WML affected only executive function and not global cognition. This finding is consistent with that of most other studies [12, 26]. It is believed that WML impairs cognition directly probably by disconnecting the 
frontosubcortical circuits, which are involved in executive control.

Our study showed that atrophy of specific frontal gyri, namely the superior frontal and fronto-orbital gyrus, predicted executive function independent of WML volume. A recent study already observed this relationship between atrophy of frontal gyri and executive dysfunction across healthy adult life span [27]. Furthermore, we found that atrophy of these frontal gyri was associated with severity of WML. Frontal gray matter atrophy correlates with aging in normal adults [27]. Our study suggests that among elderly stroke patients, concurrent WML may associate with greater frontal gray matter atrophy. Note that we could not find any association between WML and global cGM atrophy or atrophy of other cognitive eloquent cortical regions, e.g. the temporal pole, hippocampal formation, or middle occipital gyrus. Earlier studies using PET showed the preferential functional impact of WML upon frontal region, regardless of WML location $[26,28]$. Given the abundant convergence of fiber tracts on the frontal cortex from subcortical regions, we postulate that WML, if severe enough, may also induce atrophy of the frontal gray matter. Such atrophy may be due to secondary cortical structural changes, hypoperfusion, and/or hypometabolism related to subcortical white matter neuronal damage and axonal demyelination $[12,26,28]$. Our finding is similar to that of a recent study among stroke and dementia subjects, which showed that WML, in particular frontally located, was associated with atrophy of frontal gyri [29].

We also found that enlargement of the lateral ventricle predicted executive dysfunction independent of WML volume and that this ventricular enlargement was associated with the severity of WML. These findings are consistent with early studies suggesting the cognitive relevance of ventricular enlargement in lacunar patients [11] and the relationship between ventricular enlargement and WML volume [26]. Since ventricular enlargement reflects subcortical brain atrophy, it is conceivable that extensive subcortical WML might parallel subcortical brain atrophy. Overall, our findings suggest that confluent WML severity may play a central role in affecting cognition either directly by disconnecting the frontosubcortical circuits or indirectly by inducing frontal gray matter and subcortical brain atrophy.

Apart from WML severity, frontal gyri atrophy, and subcortical atrophy, we found that atrophy in the posterior limb of the internal capsule also predicted executive dysfunction. Although such a finding is novel, it is consistent with an early observation that lesion at the genu and posterior limb of the internal capsule was associated with cognitive impairment [30]. Since fibers in this defined region serve strategic reciprocal connections between frontosubcortical regions [30], we postulate that loss of fibers in this region may also impair the functional connectivity of the frontosubcortical circuits, thereby impairing executive function. However, such a hypothesis requires further investigation.

Our findings that cGM atrophy was a robust predictor of various cognitive variables independent of WML converge with previous studies [11, 12]. It has been suggested that atrophy of cGM may be a sequel common to various etiologies and induces impairment in multiple cognitive domains [12]. Although we failed to find an association between volumes of WML and cGM, as had been shown previously [12], regression analyses revealed that correlation between SBP and cGM was probably significant. Such a pattern of correlation was reported by other series as well [31]. We postulate that other hypertensive related pathologies that are not visualized by MRI, e.g. microinfarcts [32], may contribute to cGM atrophy.

We must emphasize that apart from MRI predictors, age and education also affected the cognition of our patients. This is consistent with the majority of studies showing that age and education are the most consistent risk factors for vascular cognitive impairment [33].

We failed to demonstrate any association between corpus callosum atrophy [14], multiplicity of lacunes [34], or location of lacunes (e.g. thalamic lacune) [13] with cognitive impairment as was reported by previous studies among lacunar stroke patients. Since the proportion of confluent WML among these studies was small, we postulate that these variables possibly exert a stronger cognitive influence in lacunar patients having less severe WML.

The limitations of our study include a relatively small sample size. A larger study is needed to confirm our findings. Second, since this is a cross-sectional study, the causal relationship between the putative imaging variables and cognitive impairment needs to be verified by a longitudinal study. Third, we did not determine whether hippocampal or temporal pole atrophy, which predicted the MMSE score in our study, was due to concurrent Alzheimer's pathology. Albeit this possibility, its cognitive influence was possibly negligible as the MMSE score had no impact upon patients' IADL. Last, we used a brief executive measure - the MDRS I/P - to assess executive function. Although its validity as a stand-alone executive test has been studied in over 400 elderly persons [35], a more extensive battery may pro- 
vide a fuller picture on the relationship of atrophy of various frontal gyri and its laterality to executive-behavioral functions.

A strength of our study is that we evaluated more than 100 imaging variables separately. Since investigators may bias or neglect certain imaging variables, our method would reduce such a bias as all brain regions were evaluated without prior assumption. Second, we employed an automated quantitative method for measuring WML and other brain regions, which increased the method's sensitivity and the validity of our findings.

To conclude, our findings suggest that concurrent atrophy along frontosubcortical pathways and cGM may impair executive function in lacunar patients with confluent WML. Furthermore, frontal and subcortical atrophy may be associated with the extent of WML. These findings suggest the pivotal role of WML in impairing cognition via direct and indirect influence upon frontosubcortical regions and lend support to the recent concerted efforts in retarding progression of confluent WML.

\section{Acknowledgment}

This work was supported by 2005/2006 Earmarked Grant CUHK 4317/04M.

\section{References}

-1 Roman GC, Erkinjuntti T, Wallin A, Pantoni L, Chui HC: Subcortical ischaemic vascular dementia. Lancet Neurol 2002;1:426-436.

$\checkmark 2$ Liao D, Cooper L, Cai J, et al: The prevalence and severity of white matter lesions, their relationship with age, ethnicity, gender, and cardiovascular disease risk factors: the ARIC Study. Neuroepidemiology 1997; 16:149162.

-3 Erkinjuntti T, Inzitari D, Pantoni L, et al: Research criteria for subcortical vascular dementia in clinical trials. J Neural Transm Suppl 2000;59:23-30.

4 Babikian V, Ropper AH: Binswanger's disease: a review. Stroke 1987;18:2-12.

$\checkmark 5$ Roman GC, Tatemichi TK, Erkinjuntti T, et al: Vascular dementia: diagnostic criteria for research studies. Report of the NINDS-AIREN International Workshop. Neurology 1993;43:250-260.

6 Schmidt R, Scheltens P, Erkinjuntti T, et al: White matter lesion progression: a surrogate endpoint for trials in cerebral small-vessel disease. Neurology 2004;63:139-144

7 Longstreth WT Jr, Arnold AM, Beauchamp NJ Jr, et al: Incidence, manifestations, and predictors of worsening white matter on serial cranial magnetic resonance imaging in the elderly: the Cardiovascular Health Study. Stroke 2005;36:56-61.

$>8$ Bowler JV, Gorelick PB: Advances in vascular cognitive impairment 2006. Stroke 2007; 38:241-244.

9 Fein G, Van Dyke C, Davenport L, et al: Preservation of normal cognitive functioning in elderly subjects with extensive white-matter lesions of long duration. Arch Gen Psychiatry 1990;47:220-223.

10 Yamauchi H, Fukuyama H, Ogawa M, Ouchi Y, Kimura J: Callosal atrophy in patients with lacunar infarction and extensive leukoaraiosis. An indicator of cognitive impairment. Stroke 1994;25:1788-1793.
1 Fein G, Di Sclafani V, Tanabe J, et al: Hippocampal and cortical atrophy predict dementia in subcortical ischemic vascular disease. Neurology 2000;55:1626-1635.

12 Mungas D, Jagust WJ, Reed BR, et al: MRI predictors of cognition in subcortical ischemic vascular disease and Alzheimer's disease. Neurology 2001;57:2229-2235.

13 Mok V, Chang C, Wong A, et al: Neuroimag ing determinants of cognitive performances in stroke associated with small vessel disease. J Neuroimaging 2005;15:129-137.

14 Yamauchi H, Fukuyama H, Shio H: Corpus callosum atrophy in patients with leukoaraiosis may indicate global cognitive impairment. Stroke 2000;31:1515-1520.

15 The VITATOPS(Vitamins to Prevent Stroke) Trial: Rationale and design of an international, large, simple, randomised trial of homocysteine-lowering multivitamin therapy in patients with recent transient ischaemic attack or stroke. Cerebrovasc Dis 2002;13: 120-126.

- 16 Fan YH, Lam WW, Mok VC, Huang RX, Wong KS: Variability and validity of a simple visual rating scale in grading white matter changes on magnetic resonance imaging. J Neuroimaging 2003;13:255-258.

17 Mok VCT, Wong A, Lam WWM, et al: Cognitive impairment and functional outcome after stroke associated with small vessel disease. J Neurol Neurosurg Psychiatry 2004; 75:560-566.

18 Chan AS, Choi MK, Salmon DP: The effects of age, education, and gender on the Mattis Dementia Rating Scale performance of elderly Chinese and American individuals. J Gerontol B Psychol Sci Soc Sci 2001;56: P356-P363.
19 Chiu HFK, Kee HC, Chung WS, Kwong PK: Reliability and validity of the Cantonese version of Mini-Mental State Examination - A preliminary study. J Hong Kong Coll Psychiatry 1994;4:25-28.

20 Morris JC: The Clinical Dementia Rating (CDR): current version and scoring rules. Neurology 1993;43:2412-2414.

21 Tong A, Man D: The validation of the Hong Kong Chinese version of the Lawton Instrumental Activities of Daily Living Scale for institutionalized elderly persons. Occup Ther J Res 2002;22:132-141.

22 Werring DJ, Frazer DW, Coward LJ, et al: Cognitive dysfunction in patients with cerebral microbleeds on $\mathrm{T}_{2}{ }^{*}$-weighted gradientecho MRI. Brain 2004;127:2265-2275.

23 Zhang Y, Brady M, Smith S: Segmentation of brain MR images through a hidden Markov random field model and the expectationmaximization algorithm. IEEE Trans Med Imaging 2001;20:45-57.

24 Liu T, Shen D, Davatzikos C: Deformable registration of cortical structures via hybrid volumetric and surface warping. Neuroimage 2004;22:1790-1801.

25 Liu T, Young G, Huang L, Chen NK, Wong ST: 76-space analysis of grey matter diffusivity: methods and applications. Neuroimage 2006;31:51-65.

26 DeCarli C, Murphy DG, Tranh M, et al: The effect of white matter hyperintensity volume on brain structure, cognitive performance, and cerebral metabolism of glucose in 51 healthy adults. Neurology 1995;45:20772084

27 Zimmerman ME, Brickman AM, Paul RH, et al: The relationship between frontal gray matter volume and cognition varies across the healthy adult lifespan. Am J Geriatr Psychiatry 2006;14:823-833. 
28 Tullberg M, Fletcher E, DeCarli C, et al: White matter lesions impair frontal lobe function regardless of their location. Neurology 2004;63:246-253.

29 Rossi R, Boccardi M, Sabattoli F, et al: Topographic correspondence between white matter hyperintensities and brain atrophy. J Neurol 2006;253:919-927.

30 Tatemichi TK, Desmond DW, Prohovnik I, et al: Confusion and memory loss from capsular genu infarction: a thalamocortical disconnection syndrome? Neurology 1992;42: 1966-1979.
31 Wiseman RM, Saxby BK, Burton EJ, Barber R, Ford GA, O’Brien JT: Hippocampal atrophy, whole brain volume, and white matter lesions in older hypertensive subjects. Neurology 2004;63:1892-1897.

32 Kemper T, Moss MB, Hollander W, Prusty S: Microinfarction as a result of hypertension in a primate model of cerebrovascular disease. Acta Neuropathol 1999;98:295-303.

33 Qiu C, Skoog I, Fratiglioni L: Occurrence and determinants of vascular cognitive impairment; in Erkinjuntti T, Gauthier S (eds): Vascular Cognitive Impairment. London, Dunitz, 2002, pp 61-83.
34 Corbett A, Bennett H, Kos S: Cognitive dysfunction following subcortical infarction. Arch Neurol 1994;51:999-1007.

35 Mungas D, Reed BR, Kramer JH: Psychometrically matched measures of global cognition, memory, and executive function for assessment of cognitive decline in older persons. Neuropsychology 2003;17:380392. 